\title{
Examining the Digital Story Created by an Adolescent with Cancer: Insights and Ideas from a Case Story
}

\author{
Dabney K. Wilson*, Sadie P. Hutson, Joanne M. Hall, Kim M. Anderson \\ College of Nursing, University of Tennessee, Knoxville, USA \\ Email: *dkwilson@vols.utk.edu, shutson@vols.utk.edu, jhall7@vols.utk.edu, kim.anderson@ucf.edu
}

Received 6 August 2015; accepted 28 May 2016; published 31 May 2016

Copyright (C) 2016 by authors and Scientific Research Publishing Inc.

This work is licensed under the Creative Commons Attribution International License (CC BY). http://creativecommons.org/licenses/by/4.0/

(c) (i) Open Access

\begin{abstract}
Digital Storytelling (DST) has been recognized as a holistic and innovative storytelling avenue. Survival rates for pediatric oncology patients are improved, making it necessary to investigate the psychosocial perspectives of these children. This research began following a comprehensive review of the scientific literature and identification of a gap as it pertains to using DST as an avenue for acquiring data about this aggregate of patients. The purpose of this project was to explore the use of DST among pediatric cancer patients and their perspectives regarding diagnosis. We employed a qualitative case study design with two oncology patients between the ages of $11-17$ years. The narrative, digital story, and field notes comprise the qualitative data. DST narratives and field notes were analyzed using qualitative content analysis to extract themes. Findings will provide the foundation for future research to explore the use of DST as both an intervention and data collection method in this population.
\end{abstract}

\section{Keywords}

Digital Storytelling, Adolescent, Cancer, Development, Quality of Life

\section{Introduction}

Survival rates for childhood cancers are drastically improving as medicine continues to advance [1]-[5]. As such, more children are living with cancer and the residual effects on their quality of life. Digital story is a possible avenue to gain understanding and insight into the perspectives of this unique aggregate. The Center for Digital Storytelling defines digital story as a brief film or computer-generated video, containing a combination of per-

"Corresponding author.

How to cite this paper: Wilson, D.K., Hutson, S.P., Hall, J.M. and Anderson, K.M. (2016) Examining the Digital Story Created by an Adolescent with Cancer: Insights and Ideas from a Case Story. Open Journal of Nursing, 6, 426-434.

http://dx.doi.org/10.4236/ojn.2016.65044 
sonal narrative, photographs, videos, music, and effects [6]-[8]. This study sought to investigate the role of digital storytelling in ascertaining pediatric oncology patients' perspectives regarding their cancer diagnosis and what possible therapeutic effects may be realized.

\subsection{Review of the Literature}

Throughout history, story has been a universal medium through which cultures and people groups share meaning, interactions, and memories from one generation to the next [9]-[15]. Storytelling encourages personal reflection since the elements of the story, (one's past, present, and future); must be considered [2] [6] [16]. As storytelling developed and was studied, practitioners began to recognize and use it as an instrumental element of therapy, which advanced the healing process for participants [13]. Furthermore, storytelling is recognized as a vital tool for holistic nursing care [9] [11] [17]-[20].

\subsection{Storytelling and Childhood Development}

When children tell stories, their perspective, personal orientation and relationship to a situation is revealed to the listener [9] [13]. While children's curiosity often lends them to be eager participants in storytelling, their stories have revealed beliefs that they deserve blame or are responsible for difficult situations in their life [21]. Therapeutic storytelling research requires that the listener instill a sense of autonomy and determination in these children, because it is imperative for children to understand themselves as separate from and not defined by their problems [6] [21]-[24].

For adolescents, storytelling is congruent with, and facilitates, their current stage of development. According to Erik Erikson's Theory of Human Development, adolescent children are actively seeking to understand their unique identity to avoid role confusion, and ultimately find a corresponding role in their society. Without this sense of belonging, adolescents can develop role confusion, leading to negative quality of life effects like insecurity, risky behavior, or despondence [25]-[27]. As such, adolescent children living with cancer have additional challenges in finding their identity and role in society, such as increased school absences, body image changes, struggles with peer relationships, and side effects related to treatment [28] [29]. Studies have shown that pediatric oncology patients of different ages use a variety of positive and negative coping strategies. Acknowledging the differing strategies allows for more personalized treatment plans to the patient and their current developmental level [5] [28] [30]-[32].

\subsection{Storytelling: Methods, Experiments, and Results}

Research provides support of DST as a therapeutic intervention and data collection avenue. Researchers in a three-year long study collected 566 digital stories and reported that $79 \%$ of participants stated they were either satisfied or very satisfied with the digital storytelling intervention, reporting a sense of satisfaction with the finished product and a desire to share their story with others [33]. Storytelling interventions showed improved patient-centered practice in holistic nursing care, according to Britain's National Health Service [34]. Youth programs that facilitate digital storytelling have reported themes of improved attitudes, self-esteem, cultural awareness and understanding, and a sense of achievement in the finished product [35] [36].

\subsection{Digital Storytelling}

Pediatric oncology patients form a delicate population, often unable to withstand lengthy physically or emotionally draining involvement in interventions designed to better understand their experience [4] [37]. As such, limited research and understanding of this phenomenon is found in the literature [5] [28] [34] [38] [39]. Digital storytelling presents an innovative, holistic, and plausible intervention and data collection method in which pediatric oncology patients can be better understood, cared for, and treated holistically. Digital storytelling uses a medium that is culturally and developmentally relevant to the adolescent [40] [41], and can help them develop autonomy and a sense of control and ownership over their finished product [35]. The storymaking process can further a participant's introspection into their diagnosis, as well as, provide onlookers new understandings of the pediatric cancer experience, The purpose of this study was to pilot digital storytelling as an intervention and data collection method, seeking to gain insight into the adolescent cancer experience while simultaneously evaluating for resulting quality of life changes. 


\section{Design}

This study employed a qualitative case study design, in which the participant created qualitative data through story-making. Research focused on qualitative content and narrative analysis. The data was derived from the digital story, the typed narrative, and field memos to provide a holistic and multifaceted understanding of the phenomenon studied [42]. The study was conducted over a total of ten months.

\subsection{Population and Sample}

The sample for this study was one of convenience. Adolescent patients were invited to participate in the DST process. The inclusion criteria were: age 11 to 17 years; basic computer, reading, and writing skills, ability to speak and write in English, current cancer diagnosis and undergoing treatment, willingness to participate and develop a digital story, and a signed informed assent and parental consent. The exclusion criteria centered upon the patient's functional and cognitive ability to use the technology and write and record a comprehensive personal narrative. Patients undergoing their initial round of chemotherapy treatment were excluded from the study.

Two participants in total were contacted; the first participant completed his digital story while the second participant was unable to complete the project as she began seeking treatment at another facility. The data thus is focused on a single participant, an acceptable technique in qualitative research, allowing for greater depth of analysis for this pilot study [42]. The subject was currently receiving treatment at a children's hospital in the southeastern United States.

A representative from the hospital's social work department initially approached families who met the criteria, briefly explained the study, and assessed their level of interest. The Principal Investigator (PI) was then invited to meet with interested families individually to explain the digital storytelling process and obtain informed consent and child assent. The parents of possible participants reviewed inclusion criteria, project purpose, risks and benefits, confidentiality, and right to withdraw at any time. Following signature of the parental consent, informed pediatric assent was obtained.

\subsection{Data Collection}

Before beginning data collection, the PI completed a bracketing interview to identify and limit sources of bias. The PI then worked with the participant during three separate chemotherapy treatment cycles to elicit a narrative draft of his story. Discussing pictures, video, and/or music that he wished to include in the digital story aided in writing the narrative portion (Lambert, 2010). The investigator took extensive field memos to record observations about the process during the participant's treatment, including contextual notes. Working with the participant during treatment provided several hours of time in a safe, quiet, and private environment.

The tools used in this study were to implement the intervention (making the digital story), and to examine the related experience. The PI and the participant collaboratively created the digital story using a storyboard and story-lining worksheet to produce the written narrative. The participant used an iPad with iMovie to compile the chosen elements of his story. The participant also completed a brief demographic inventory.

The three sessions were based upon the following schedule of activities. 1) Session one included general introductions, introducing the basic concepts of storytelling, including using a storyboard to begin to lay out which elements of his story he wished to tell (see Figure 1). 2) Session two consisted of story-lining and writing the narrative portion (see Figure 2). Next, he recorded his narrative on the iPad using the narration feature in iMovie. The researcher briefly ( $<30$ minutes) introduced how to use iMovie software. Then the participant practiced and became comfortable with using the technology. Between sessions the participant collected images and sounds to insert into the narrative. 3) The focus of session three was to integrate all of the information together into the finished digital story. Sessions remained flexible, allowing the participant to determine the pace, adjusting to best reach the participant's desired digital story. Each session lasted between 60 and 120 minutes.

\subsection{Human Subjects Considerations}

The study had full review of the Institutional Review Boards at the data collection site and at the University. The possible benefits of this research, for the individual participant as well as for extending the knowledge base, included improved: quality of life, socialization and/or family bond, intrapersonal relationship, and understanding of the adolescent cancer experience. Finally, the researcher sought to expand the current understanding of this 

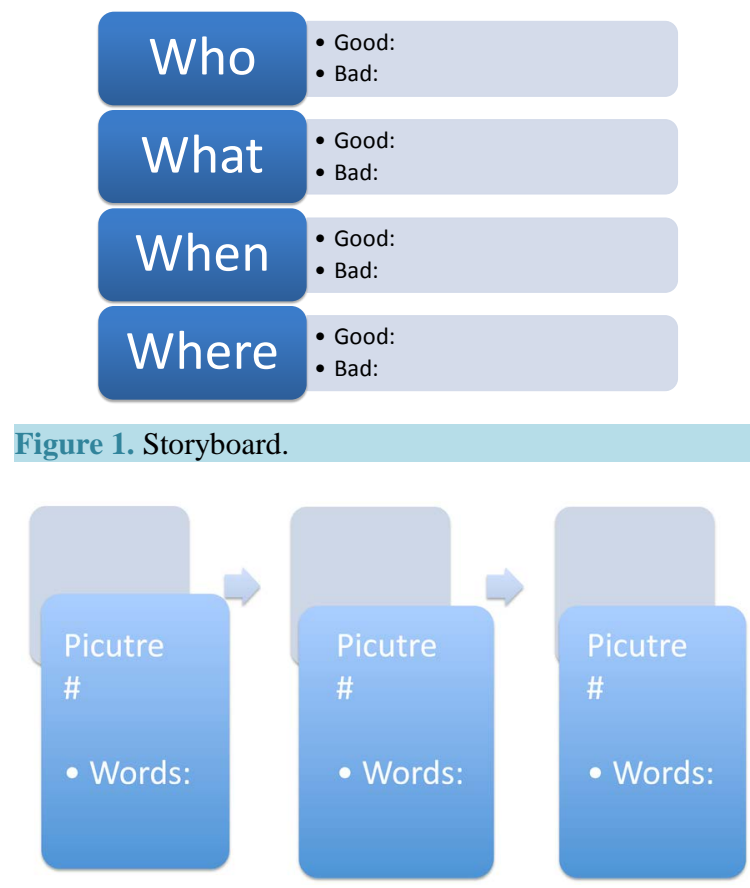

Figure 2. Storyline.

group of patients to improve care and health outcomes for future patients. This research should be considered a pilot for future exploration and development of interventions implementing DST into holistic patient care.

The potential risks of participating in this research study included personal unrest and frustration during the storytelling process, use of somewhat complex technology, and possible fatigue. There were no other foreseeable risks; risks were minimized through close supervision of the charge nurse and the hospital's child well-being program throughout and/or after each session as needed.

\subsection{Data Analysis}

In order to create a complete description of the phenomenon, all data was analyzed using a combination of qualitative content analysis and narrative analysis [43]-[46]. These strategies identified common themes and individual characteristics about the patient's perspective toward diagnosis and the process of creating a digital story.

Investigators reviewed the digital story multiple times. First, each investigator explored the story as a whole. This formed a tentative overall message. The data from each close reading was then examined and the analysis from each investigator was compared and discussed to accommodate diversity, and to promote consistency and dependability in interpretation [2] [42] [43]. After close readings, meaningful phrases were identified in the narratives and the field notes. These phrases or words were then coded and categorized. Next, the media images, pictures, etc, were combined with the categories derived from the textual narrative data. These categories were then condensed into four themes. Thus, the themes were based on the narrative, pictures, music, and voice inflection [43] presented in the digital story, as well as the field notes [2]. Each of the themes is presented in detail in the Results section.

\section{Results}

\subsection{Participants}

The participant was a 17-year-old male who had responded to recruitment, been diagnosed within 3 - 6 months, and consented to participate in the study. He was familiar with using a computer, iPad, or tablet for more than 7 years. He completed all study procedures, resulting in a 2:11 minute digital story made up of a 351-word spoken narrative, 29 pictures, written captions, and an instrumental song. The digital story depicted the participant's experience leading up to and following his diagnosis with cancer. 


\subsection{Data}

Data consisted of four main themes derived from the digital story and researcher written field notes, as shown in Figure 3 [42]. Although the finished product functioned as one fluid story, four themes emerged and were further analyzed to better understand the phenomenon of making a digital story. Illustrative quotes provided herein support the study themes. The four main data-driven themes were: 1) normalization of the cancer experience, 2) disruption caused by a cancer diagnosis, 3) uncertainty about the future, and 4) formation of a testimony amidst change.

Throughout the digital story, the participant sought to normalize his experience, drawing parallels between his life before and after his diagnosis. He incorporated objects associated with his treatment into age-appropriate activities and life experiences, such as driving and meeting girls. The digital story's climax was a disruption to this seemingly normal teenage life: being told of his diagnosis. The narrative form after the climax trended downward; it descended into images and words about his routine events, and about losses associated with this climactic disruption.

The imagery chosen for the climactic moment of the narrative was that of a screeching halt cartoon, with no personal photographs, feelings, or thoughts shared. There was a strong sense of disconnect created in this moment, as the diagnosis seemed to be thrust upon him, lacking ownership. The uncertainty of the future was part of the descent, as indicated by the prevalence of these thoughts into his current story. Finally, there was an upward turn, resembling a testimonial in which he developed a theme of newfound inner strength as one result of the diagnosis. Throughout he mentions few people, but as he discussed positives, he talked of the support system found in his religious community. Imagery in the narrative included that of a battlefield, as he fought against the enemy of cancer. This testimony translated a significant sense of hope to the audience.

Disruption caused by the cancer diagnosis. The disruption was the central event within the narrative as well a theme throughout the resulting story. The participant built up to the moment his diagnosis was determined, and in that moment, his tense cadence, and audible tone shift reflected a disconnect. He stated, "I was told I have leukemia” and to illustrate he chose an impersonal picture of a well-known cartoon character suddenly stopping an automobile.

Uncertainty about the future. The uncertainty for the future was a theme developed at both the beginning and end of the digital story. The participant had begun discussing his view before his diagnosis, describing it as "full of hopes and dreams for the future." He asked, presumably rhetorically, at the end, "How does the story end? The ending is not written yet," and "where do I hope to be in six months?" There was a shift in how he discussed this uncertainty after the diagnosis was revealed, acknowledging the loss of predictability in life due to, and since his diagnosis.

Normalization of the cancer experience. His story clearly contrasted normal daily life before and after the cancer diagnosis. He first described his mundane, usual high school life. He then detailed the happenings and

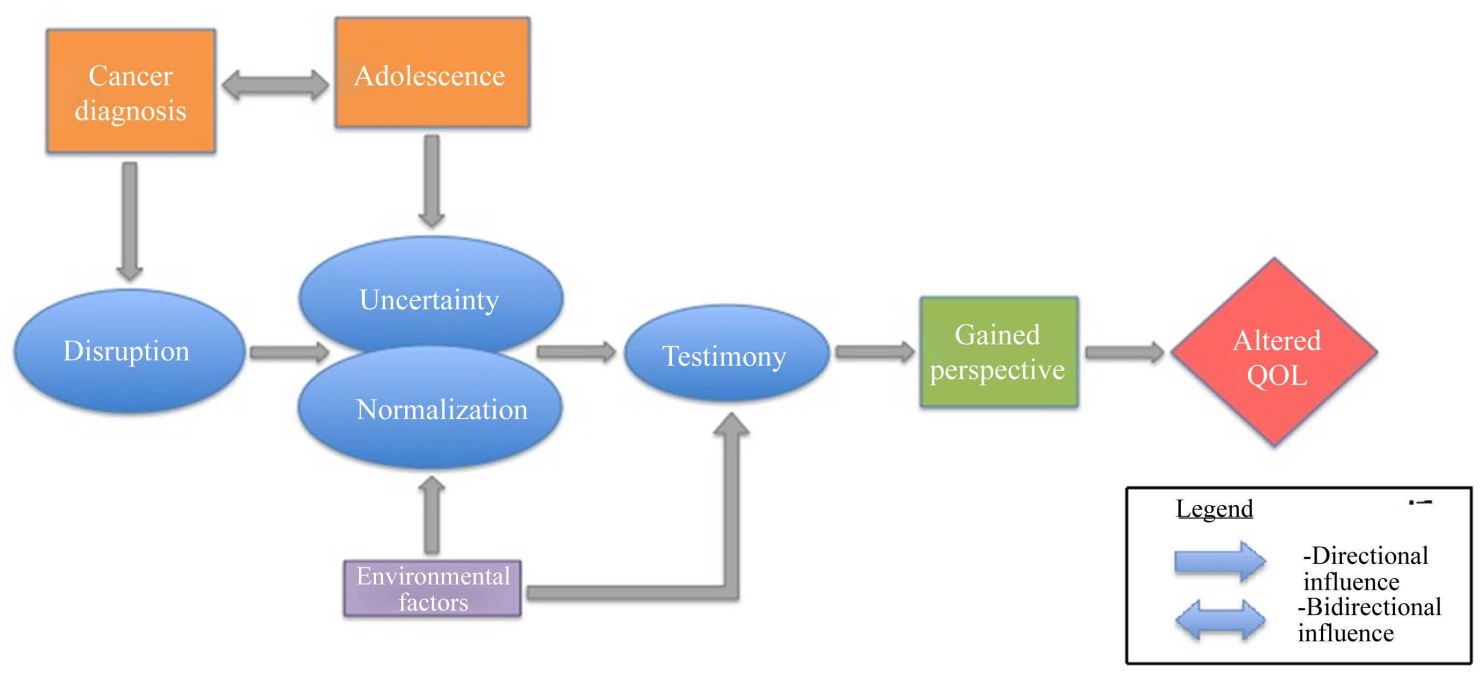

Figure 3. Primary findings. 
adaptations made after diagnosis to a newly established normal routine. One specific incidence discussed was a possible new use for his radiotherapy mask. He stated, "then there's radiation, there's nothing cool about thatexcept for the awesome mask that I am going to be able to wear next Halloween.” He discussed taking this object that was isolated to the cancer experience and assimilating it into a normal adolescent activity (wearing it as a costume for Halloween).

Testimony amidst change. The development of a testimony amidst change was a theme encompassing his altered social roles, personal spirituality, and increased awareness of his support system. This theme was supported by the photographs in the digital story. Each image accompanying this section of the narrative was a personal photograph of cards and gifts given, banners painted, and people who have gathered in his support. Socially, he identifies himself as "the guy with the cool hair" before diagnosis and then he transitions to an image of the "cool" hats he now wears on his bare head, a testimony to his ability to adapt.

\subsection{Discussion}

The findings of this study affirmed the usability and adaptability of digital storytelling as a communication intervention with an adolescent oncology patient. The results provide previously unseen insight into the adolescent's perspective of the cancer diagnosis and experience. Results were consistent with Erik Erikson's Theory of Human Development for the adolescent, focused on identity and social belongingness with peers. Notably absent was mention of his family or any other named characters or their influence in his story, presumably an attempt to develop a theory of self, independent from parental influence. Similarly, he displayed limited emotionality, choosing rather to discuss the daily happenings of his life, likely in an effort to normalize his circumstances to his expected level of independence and social standing. Specifically, when discussing the uncertainty of his future, he referred to his education and career, consistent with the search for an avenue where he can significantly contribute to society. Finally, he acknowledged the junction of his past and future, consistent with Erikson’s projections. He stated that he has learned "that I am stronger than I thought” while also stating that his ordinary life is lost to the uncertain future.

The syntactical structure of the digital story was circular, beginning with a discussion of hope for the future, peaking at the moment of diagnosis, descending into the resulting deeper levels of personal loss. He then transitioned into a newfound testimony that finally ended in the same hope for the future that began the journey into his story.

Furthermore, there is a consistently casual tone, conveying his story as a journey shared with the audience. He used personal possessive pronouns, often shifting from past to present and future tense, as if the audience was listening to his internal dialogue. He began by addressing how "I never thought I would have to spend my senior year hanging out here [the hospital] everyday," and shifted at the end of the narrative to contemplating how he hopes his senior year will end. This easy-going tone is amplified as he indirectly addressed the audience in a playful manner four different times throughout, asking rhetorical questions and using plural pronouns.

Overall, the data suggests the benefit and usefulness of a digital storytelling intervention among this population, although more research is needed. Additional studies with more participants to validate this approach and gain additional understanding of the lived experience of adolescents with cancer can lead to improved care for healthcare providers for this unique population.

\subsection{Limitations}

Even though qualitative studies typically use smaller sample sizes, this study was limited to one subject completing the project. More subjects were sought but not recruited. This population presents a challenge as nature of treatment schedules were frequently interrupted by complications, most often neutropenia (e.g., making it difficult to schedule future session appointments due to rescheduled chemotherapy treatments). Confounding factors also limited the data as no control measures or efforts were made to minimize or analyze factors such as the role of the family, time since diagnosis, or cultural influences.

\subsection{Implications}

Several implications for practice arose from this project, including: learning to openly acknowledge and empathize with the uncertainty of cancer for the adolescent, refraining from assuming a support system (e.g., pa- 
rental), and recognizing the value of digital storytelling as both a therapeutic intervention and data collection method for this vulnerable population.

Data from this pilot project suggested that the participant was acutely aware that cancer alters his future from what is expected in his developmental stage. Recognizing this understanding provides opportunity for open communication that can provide support rather than disregard the reality of a cancer diagnosis for the adolescent. Furthermore, his story revealed that his reference group and support system was primarily his peers, without mention of family. Healthcare workers must resist the urge to assume reliance upon a traditional parental support system, a bias found in cultures where a mother-father dyad are thought to be the norm. When the patient does not come from a traditional home or does not choose to find his support system there, projecting this could cause him to feel further ostracized from a normal adolescent experience. Finally, this single case analysis serves as a foundation for future research. Since the participant successfully constructed his digital story using the technology provided, this intervention could be tested and adapted, if needed, in persons of other developmental levels and diagnoses to attain a more complete picture of the phenomenon and the resulting change in quality of life after the DST intervention.

Implications for future studies implementing a digital storytelling intervention with the pediatric oncology population would benefit from studying the compounding factors, such as family, as well as the recipients of the finished digital story. Finally, a large study sample across differing cultures would better reflect the usefulness of this intervention and analysis of the process and the final created product.

\section{Conclusion}

The significance of story has transcended generations and cultures and is now well documented as both a therapeutic agent and method for data collection in the literature. More recently, digital storytelling has been used to attain these results. This study implemented digital storytelling with a young male individual from a patient population that is often isolated due to the barriers posed by diagnosis and treatment of cancer. The adolescent with cancer has proven to be a successful storyteller, improving both his quality of life as well as providing valuable insight to better care for and understand this patient population. Therapeutic as well as empirical benefits are available in digital storytelling research that is consistent with nursing's commitment to aesthetic knowledge, development, and holistic treatment.

\section{Acknowledgements}

The first author would like to acknowledge the Chancellor's Honors Program and the Nursing Honors Program at the University of Tennessee-Knoxville.

\section{References}

[1] de Vries, M.C., Houtlosser, M., Wit, J.M., et al. (2011) Ethical Issues at the Interface of Clinical Care and Research Practice in Pediatric Oncology: A Narrative Review of Parents’ and Physicians’ Experiences. BMC Medical Ethics, 12, 18. http://dx.doi.org/10.1186/1472-6939-12-18

[2] Enskar, K. (2012) Being an Expert Nurse in Pediatric Oncology Care: Nurses’ Descriptions in Narratives. Journal of Pediatric Oncology Nursing: Official Journal of the Association of Pediatric Oncology Nurses, 29, 151-160. http://dx.doi.org/10.1177/1043454212446344

[3] Klassen, A.F., Anthony, S.J., Khan, A., Sung, L. and Klaassen, R. (2011) Identifying Determinants of Quality of Life of Children with Cancer and Childhood Cancer Survivors: A Systematic Review. Supportive Care in Cancer: Official Journal of the Multinational Association of Supportive Care in Cancer, 19, 1275-1287. http://dx.doi.org/10.1007/s00520-011-1193-x

[4] Stutzer, C.A., Drew, D., Himelstein, B.P., et al. (2005) Consensus Statement: Collaborative Clinical Research on Endof-Life Care in Pediatric Oncology. Seminars in Oncology Nursing, 21, 142-144. http://dx.doi.org/10.1016/j.soncn.2004.12.014

[5] Wu, L.M., Chin, C.C., Haase, J.E. and Chen, C.H. (2009) Coping Experiences of Adolescents with Cancer: A Qualitative Study. Journal of Advanced Nursing, 65, 2358-2366. http://dx.doi.org/10.1111/j.1365-2648.2009.05097.x

[6] Anderson, K.M. (2010) Enhancing Resilience in Survivors of Family Violence. Springer Publishing Company, LLC, New York, 99-116.

[7] Jernigan, V.B., Salvatore, A.L., Styne, D.M. and Winkleby, M. (2012) Addressing Food Insecurity in a Native Ameri- 
can Reservation Using Community-Based Participatory Research. Health Education Research, 27, 645-655. http://dx.doi.org/10.1093/her/cyr089

[8] Lambert, J. (2010) Digital Storytelling Cookbook. Digital Diner Press.

[9] Backhaus, K.A. (1984) Life Books: Tools for Working with Children in Placement. Social Work, 29, 551-554.

[10] Badger, K., Royse, D. and Moore, K. (2011) What's in a Story? A Text Analysis of Burn Survivors' Web-Posted Narratives. Social Work in Health Care, 50, 577-594. http://dx.doi.org/10.1080/00981389.2011.592114

[11] Banks-Wallace, J. (1999) Story Telling as a Tool for Providing Holistic Care to Women. The American Journal of Maternal Child Nursing, 24, 20-24. http://dx.doi.org/10.1097/00005721-199901000-00005

[12] Larkey, L.K., Lopez, A.M., Minnal, A. and Gonzalez, J. (2009) Storytelling for Promoting Colorectal Cancer Screening among Underserved Latina Women: A Randomized Pilot Study. Cancer, Culture and Literacy, 16, 79-87.

[13] Scaletti, R. and Hocking, C. (2010) Healing through Story Telling: An Integrated Approach for Children Experiencing Grief and Loss. New Zealand Journal of Occupational Therapy, 57, 66-71.

[14] Larkey, L.K. and Gonzalez, J. (2007) Storytelling for Promoting Colorectal Cancer Prevention and Early Detection among Latinos. Patient Education and Counseling, 67, 272-278. http://dx.doi.org/10.1016/j.pec.2007.04.003

[15] Polkinghorne, D.E. (1996) Transformative Narratives: From Victimic to Agentic Life Plots. American Journal of Occupational Therapy, 50, 299-305. http://dx.doi.org/10.5014/ajot.50.4.299

[16] Gilbert, K.R. (2002) Taking a Narrative Approach to Grief Research: Finding Meaning in Stories. Death Studies, 26, 223-239. http://dx.doi.org/10.1080/07481180211274

[17] Buttery, J., Eades, M., Frisch, S., Giguere, M. and Mountjoy, A. (1999) Family Response to Difficult Hospitalizations: The Phenomenon of "Working through". Journal of Clinical Nursing, 8, 459-466. http://dx.doi.org/10.1046/j.1365-2702.1999.00257.x

[18] Chelf, J.H., Deshler, A.M., Hillman, S. and Durazo-Arvizu, R. (2000) Storytelling: A Strategy for Living and Coping with Cancer. Cancer Nursing, 23, 1-5. http://dx.doi.org/10.1097/00002820-200002000-00001

[19] Smith, M.J. and Liehr, P. (1999) Attentively Embracing Story: A Middle-Range Theory with Practice and Research Implications. Scholarly Inquiry for Nursing Practice, 13, 187-204, Discussion 5-10.

[20] Smith, M.J. and Liehr, P.R. (2014) Middle Range Theory for Nursing. 3rd Edition, Springer Publishing Company, New York.

[21] Furlonger, B.E. (1999) Narrative Therapy and Children with Hearing Impairments. American Annals of the Deaf, 144, 325-333. http://dx.doi.org/10.1353/aad.2012.0311

[22] Matos, M., Santos, A., Goncalves, M. and Martins, C. (2009) Innovative Moments and Change in Narrative Therapy. Psychotherapy Research, 19, 68-80. http://dx.doi.org/10.1080/10503300802430657

[23] McCarthy, P.G. and Sebaugh, J.G. (2011) Therapeutic Scrapbooking: A Technique to Promote Positive Coping and Emotional Strength in Parents of Pediatric Oncology Patients. Journal of Psychosocial Oncology, 29, 215-230. http://dx.doi.org/10.1080/07347332.2010.548443

[24] Wallis, J., Burns, J. and Capdevila, R. (2011) What Is Narrative Therapy and What Is It Not? The Usefulness of Q Methodology to Explore Accounts of White and Epston's (1990) Approach to Narrative Therapy. Clinical Psychology \& Psychotherapy, 18, 486-497. http://dx.doi.org/10.1002/cpp.723

[25] Erikson, E.H. (1959) Identity and the Life Cycle. International Universities Press Inc., New York.

[26] Erikson, E.H. (1963) Childhood and Society. Norton, New York.

[27] Kishton, J.M. (1994) Contemporary Eriksonian Theory: A Psychobiographical Illustration. Gerontology \& Geriatrics Education, 14, 81-91. http://dx.doi.org/10.1300/J021v14n04_07

[28] Hildenbrand, A.K., Clawson, K.J., Alderfer, M.A. and Marsac, M.L. (2011) Coping with Pediatric Cancer: Strategies Employed by Children and Their Parents to Manage Cancer-Related Stressors during Treatment. Journal of Pediatric Oncology Nursing: Official Journal of the Association of Pediatric Oncology Nurses, 28, 344-354. http://dx.doi.org/10.1177/1043454211430823

[29] Pini, S., Hugh-Jones, S. and Gardner, P.H. (2012) What Effect Does a Cancer Diagnosis Have on the Educational Engagement and School Life of Teenagers? A Systematic Review. Psycho-Oncology, 21, 685-694. http://dx.doi.org/10.1002/pon.2082

[30] Li, H.C., Chung, O.K., Eva Ho, K.Y., Chiu, S.Y. and Lopez, V. (2011) Coping Strategies Used by Children Hospitalized with Cancer: An Exploratory Study. Psycho-Oncology, 20, 969-976.

[31] Rodgers, C., Norville, R., Taylor, O., et al. (2012) Children’s Coping Strategies for Chemotherapy-Induced Nausea and Vomiting. Oncology Nursing Forum, 39, 202-209. http://dx.doi.org/10.1188/12.ONF.202-209

[32] Engvall, G., Mattsson, E., von Essen, L. and Hedstrom, M. (2011) Findings on How Adolescents Cope with Cancer- 
A Matter of Methodology? Psycho-oncology, 20, 1053-1060. http://dx.doi.org/10.1002/pon.1809

[33] Wexler, L., Gubrium, A., Griffin, M. and Difulvio, G. (2012) Promoting Positive Youth Development and Highlighting Reasons for Living in Northwest Alaska through Digital Storytelling. Health Promotion Practice, 14, 617-623.

[34] Clarke, A., Hanson, E.J. and Ross, H. (2003) Seeing the Person behind the Patient: Enhancing the Care of Older People Using a Biographical Approach. Journal of Clinical Nursing, 12, 697-706. http://dx.doi.org/10.1046/j.1365-2702.2003.00784.x

[35] Hull, G.A. and Katz, M.-L. (2006) Crafting an Agentive Self: Case Studies of Digital Storytelling. Research in the Teaching of English, 41, 43-81.

[36] Davis, A. and Weinshenker, D. (2012) Digital Storytelling and Aurthoring Identity. In: Ching, C.C. and Foley, B., Eds., Technology and Identity: Research on the Development and Exploration of Selves in a Digital World, Cambridge University Press, Cambridge, 47-64.

[37] Ruland, C.M., Starren, J. and Vatne, T.M. (2008) Participatory Design with Children in the Development of a Support System for Patient-Centered Care in Pediatric Oncology. Journal of Biomedical Informatics, 41, 624-635. http://dx.doi.org/10.1016/j.jbi.2007.10.004

[38] Peterson, C.C., Cousino, M.K., Donohue, J.E., Schmidt, M.L. and Gurney, J.G. (2012) Discordant Parent Reports of Family Functioning Following Childhood Neuroblastoma: A Report from the Children's Oncology Group. Journal of Psychosocial Oncology, 30, 503-518. http://dx.doi.org/10.1080/07347332.2012.703766

[39] Williams-Brown, S., Baldwin, D.M. and Bakos, A. (2002) Storytelling as a Method to Teach African American Women Breast Health Information. Journal of Cancer Education: The Official Journal of the American Association for Cancer Education, 17, 227-230.

[40] Davis, A. (2004) Co-Authoring Identity: Digital Storytelling in an Urban Middle School. THEN: Technology, Humanities, Education and Narrative, 1, 1-12.

[41] Sawyer, C.B. and Willis, J.M. (2011) Introducing Digital Storytelling to Influence the Behavior of Children and Adolescents. Journal of Creativity in Mental Health, 6, 274-283. http://dx.doi.org/10.1080/15401383.2011.630308

[42] Baxter, P. and Jack, S. (2008) Qualitative Case Study Methodology: Study Design and Implementation for Novice Researchers. The Qualitative Report, 13, 544-559.

[43] Graneheim, U.H. and Lundman, B. (2004) Qualitative Content Analysis in Nursing Research: Concepts, Procedures and Measures to Achieve Trustworthiness. Nurse Education Today, 24, 105-112. http://dx.doi.org/10.1016/j.nedt.2003.10.001

[44] Sandelowski, M. (1994) We Are the Stories We Tell: Narrative Knowing in Nursing Practice. Journal of Holistic Nursing: Official Journal of the American Holistic Nurses' Association, 12, 23-33. http://dx.doi.org/10.1177/089801019401200105

[45] Sandelowski, M. (2000) Whatever Happened to Qualitative Description? Research in Nursing \& Health, 23, $334-340$. http://dx.doi.org/10.1002/1098-240X(200008)23:4<334::AID-NUR9>3.0.CO;2-G

[46] Sandelowski, M. (2010) What's in a Name? Qualitative Description Revisited. Research in Nursing \& Health, 33, 7784. 